Homology, Homotopy and Applications, vol.16(2), 2014, pp.33-43

\title{
HOMOLOGICAL DESCENT FOR MOTIVIC HOMOLOGY THEORIES
}

\author{
THOMAS GEISSER
}

(communicated by Charles A. Weibel)

\begin{abstract}
The purpose of this paper is to give homological descent theorems for motivic homology theories (for example, Suslin homology) and motivic Borel-Moore homology theories (for example, higher Chow groups) for certain hypercoverings.
\end{abstract}

\section{Introduction}

Let $S$ be a separated, noetherian scheme of finite Krull dimension, and let $\mathcal{F}$ be a covariant functor from the category of schemes separated and of finite type over $S$ and proper morphisms to the category of (homologically) positive complexes of abelian groups with $\mathcal{F}(\emptyset)=0$. We assume that for every abstract blow-up square, there is a distinguished triangle

$$
\mathcal{F}\left(Z^{\prime}\right) \rightarrow \mathcal{F}\left(X^{\prime}\right) \oplus \mathcal{F}(Z) \rightarrow \mathcal{F}(X) \rightarrow \mathcal{F}\left(Z^{\prime}\right)[1]
$$

in the derived category of abelian groups. Here an abstract blow-up square is a diagram of the form

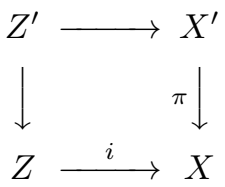

with $\pi$ proper, $i$ a closed embedding, and such that $\pi$ induces an isomorphism $X^{\prime}-Z^{\prime} \rightarrow X-Z$. The morphism $Z \amalg X^{\prime} \rightarrow X$ is then called an abstract blow-up. In particular (taking $X^{\prime}=\emptyset$ ), every closed embedding $Z \rightarrow X$ defined by a nilpotent ideal includes a quasi-isomorphism $\mathcal{F}(Z) \rightarrow \mathcal{F}(X)$.

Recall that a proper cdh-cover is a proper map $p: X^{\prime} \rightarrow X$ such for every point $x \in X$, there is a point $x^{\prime} \in X^{\prime}$ with $p\left(x^{\prime}\right)=x$ and $p^{*}: k(x) \stackrel{\sim}{\rightarrow} k\left(x^{\prime}\right)$, and that a hyperenvelope is an augmented simplicial scheme $a: X_{\bullet} \rightarrow X$ such that, for every $n$, the map $X_{n+1} \rightarrow\left(\operatorname{cosk}_{n} X_{\bullet}\right)_{n+1}$ is a proper cdh-cover. For a simplicial scheme $X_{\bullet}$, we simply write $\mathcal{F}\left(X_{\bullet}\right)$ for the total complex of the simplicial complex of abelian groups.

Supported by JSPS Grant 23340004

Received January 30, 2014, revised April 18, 2014; published on July 2, 2014.

2010 Mathematics Subject Classification: 14F42, $19 \mathrm{E} 15$.

Key words and phrases: Descent, hypercovering, motivic homology theory.

Article available at http://dx.doi.org/10.4310/HHA.2014.v16.n2.a2

Copyright (C) 2014, International Press. Permission to copy for private use granted. 
Theorem 1.1. For any functor as above, and for any hyperenvelope $a: X \bullet \rightarrow X$, the augmentation map induces a quasi-isomorphism

$$
\mathcal{F}\left(X_{\bullet}\right) \rightarrow \mathcal{F}(X)
$$

In characteristic $p$, smooth hyperenvelopes are only known to exist under resolution of singularities. To remove this hypothesis, and to be able to use Gabber's refinement of de Jong's theorem on alterations, we consider l-hyperenvelopes. A proper ldh-cover is a proper surjection $p: X^{\prime} \rightarrow X$ such that for every point $x \in X$ there is a point $x^{\prime} \in X^{\prime}$ with $p\left(x^{\prime}\right)=x$, and such that $k\left(x^{\prime}\right)$ is a finite extension of degree prime to $l$ of $p^{*}(k(x))$. An $l$-hyperenvelope is an augmented simplicial scheme $a: X \bullet \rightarrow X$ such that $X_{n+1} \rightarrow\left(\operatorname{cosk}_{n} X_{\bullet}\right)_{n+1}$ is a proper ldh-cover for all $n$.

Theorem 1.2. Assume that $\mathcal{F}$ is a functor to the category of complexes of $\mathbb{Z}_{(l)}{ }^{-}$ modules, which satisfies the following property in addition to the above:

For any finite flat map $p: X \rightarrow Y$ of degree d prime to $l$, there is a functorial pullback map $p^{*}: \mathcal{F}(Y) \rightarrow \mathcal{F}(X)$ such that $p_{*} p^{*}$ induces multiplication by d on homology, and which is compatible with base-change.

Then for any l-hyperenvelope $a: X \bullet \rightarrow X$, the augmentation map induces a quasiisomorphism

$$
\mathcal{F}\left(X_{\bullet}\right) \rightarrow \mathcal{F}(X)
$$

One can see that some hypothesis on the coefficients is necessary by considering the Cech-nerve $\operatorname{cosk}_{0}(L / k)$ of a finite field extension $L / k$ of degree $d$ : The map $H_{0}^{S}(L, \mathbb{Z}) \rightarrow H_{0}^{S}(k, \mathbb{Z})$ is multiplication by $d$ on $\mathbb{Z}$, hence descent does not hold.

The proof of the theorems is along the same lines as [16, §3]. Gillet [8], and Gillet-Soulé [9] used a similar argument to prove descent for theories satisfying the localization property (like higher Chow groups and $K^{\prime}$-homology), but, using the notes of B. Conrad [2], we give a self-contained proof that, in addition, does not require the localization property.

As an application, we obtain the following descent theorem for the motivic homology groups $H_{i}(X, A(n))$, motivic Borel-Moore homology groups $H_{i}^{B M}(X, A(n))$, and higher Chow groups:

Theorem 1.3. Let $X$ be a separated scheme of finite type over a perfect field, and $A$ be an abelian group. Suppose that $a: X_{\bullet} \rightarrow X$ is a hyperenvelope and resolution of singularities holds, or that $a: X \bullet \rightarrow X$ is a l-hyperenvelope and that $A$ is a $\mathbb{Z}_{(l)}{ }^{-}$ module. Then we have spectral sequences

$$
\begin{aligned}
E_{p, q}^{1}=H_{q}\left(X_{p}, A(r)\right) & \Rightarrow H_{p+q}(X, A(r)), \\
E_{p, q}^{1}=H_{q}^{B M}\left(X_{p}, A(r)\right) & \Rightarrow H_{p+q}^{B M}(X, A(r)) .
\end{aligned}
$$

For a separated scheme of finite type over a Dedekind ring, we define higher Chow groups as the Zariski hypercohomology of Bloch's cycle complex (for schemes over fields, this agrees with the homology of the global sectons of the cycle complex).

Theorem 1.4. Let $X$ be separated and of finite type over a field or a Dedekind ring, and $A$ be an abelian group. Suppose that $a: X \bullet \rightarrow X$ is a hyperenvelope, or that $A$ is 
$a \mathbb{Z}_{(l)}$-module and $a: X \bullet \rightarrow X$ an l-hyperenvelope. Then we have spectral sequences for any $n \in \mathbb{Z}$,

$$
E_{p, q}^{1}=C H_{n}\left(X_{p}, q, A\right) \Rightarrow C H_{n}(X, p+q, A) .
$$

The analogous result for $K^{\prime}$-theory can be proven by the same method.

We note that $l$-hyperenvelopes exist by by a theorem of Gabber:

Theorem 1.5. For every separated scheme $U$ of finite type over a field $k$, and for every $l \neq$ char $k$, there exists an l-hyperenvelope $U_{\bullet} \rightarrow U$ such that $U_{\bullet}$ is an open simplicial subscheme of a simplicial scheme consisting of smooth projective schemes over $k$.

\section{Acknowledgments}

We thank S. Kelly for helpful comments, discussions, and explanation of his work, and the referee for additional helpful comments.

\section{Simplicial schemes and hyperenvelopes}

Let $\mathcal{C}$ be a category with finite limits. A simplicial object in $\mathcal{C}$ is a functor $X_{\bullet}: \Delta^{\mathrm{op}} \rightarrow$ $\mathcal{C}$, where $\Delta$ is the simplicial category of finite ordered sets $[i]=\{0, \ldots, i\}$ with nondecreasing maps. As usual, we write $X_{n}$ for $X_{\bullet}([n])$ and $\alpha^{*}: X_{j} \rightarrow X_{i}$ instead of $X_{\bullet}(\alpha)$ for $\alpha:[i] \rightarrow[j]$. If $\Delta_{\leqslant n}$ is the full subcategory of $\Delta$ consisting of $[0], \ldots,[n]$, then the restriction functor $i_{n}^{*}$ from simplicial objects to restricted simplicial objects (i.e., functors $\Delta_{\leqslant n}^{\mathrm{op}} \rightarrow \mathcal{C}$ ) has a left adjoint skeleton $\mathrm{sk}_{n}$ and a right adjoint coskeleton $\left(i_{n}\right)_{*}=\operatorname{cosk}_{n}$. We note that in the literature, the notation $\mathrm{sk}_{n}$ appears both as the name of the restriction functor [3] and as the name of its left adjoint (e.g., SGA $4 \mathrm{~V} 7$ ). By abuse of notation, we also denote the composition of $\left(i_{n}\right)_{*} i_{n}^{*}$ by $\operatorname{cosk}_{n}$. In this notation, the adjunction map takes the form $X_{\bullet} \rightarrow \operatorname{cosk}_{n} X_{\bullet}$. Concretely,

$$
\left(\operatorname{cosk}_{n} X_{\bullet}\right)_{m}=\lim _{D_{m}^{\prime}} X_{\phi},
$$

where $D_{m}^{\prime}$ is the category of non-decreasing maps $\phi:[i] \rightarrow[m]$ with $i \leqslant n, X_{\phi}=X_{i}$, with morphisms $\alpha: \phi \rightarrow \phi^{\prime}$ those non-decreasing maps $[i] \rightarrow\left[i^{\prime}\right]$ that are compatible with the maps to $[m]$. This can also be expressed as follows [2, Cor. 3.10]: Let $D_{m}$ be the full subcategory of $D_{m}^{\prime}$ with objects increasing injections $\phi:[i] \rightarrow[m]$ for $i \leqslant n$ (which implies that the morphisms $\alpha: \phi \rightarrow \phi^{\prime}$ are also injective). Then $\left(\operatorname{cosk}_{n} X_{\bullet}\right)_{m}=$ $X_{m}$ for $m \leqslant n$ and, for $m>n,\left(\operatorname{cosk}_{n} X_{\bullet}\right)_{m}$ is the equalizer of the maps

$$
s, t: \prod_{\phi \in o b D_{m}} X_{\phi} \rightarrow \prod_{\alpha \in \operatorname{mor} D_{m}} X_{\alpha}
$$

where $X_{\alpha}=X_{\phi}$ for $\alpha:[\phi] \rightarrow\left[\phi^{\prime}\right]$, and on the component indexed by $\alpha, s$ is the projection to $X_{\phi}$, whereas $t$ is the projection to $X_{\phi^{\prime}}$ followed by $\alpha^{*}$. In particular, we obtain

$$
\operatorname{cosk}_{n} \stackrel{\sim}{\longrightarrow} \operatorname{cosk}_{n} \operatorname{cosk}_{m}
$$

for $n \leqslant m$. Similarly, for $n \leqslant m$, the restriction functor $\left(i_{n}^{m}\right)^{*}$ from $m$-truncated simplicial sets to $n$-truncated simplicial sets has a left adjoint and a right adjoint $\left(i_{n}^{m}\right)_{*}$. 
If $n \leqslant m$, then

$$
\operatorname{cosk}_{n} \stackrel{\sim}{\longrightarrow} \operatorname{cosk}_{m} \operatorname{cosk}_{n}
$$

(this is wrongly stated in SGA $4 \mathrm{~V}$ 7.1.2). Indeed, we just apply the following to $i_{n}^{*}$ :

$$
\left(i_{n}\right)_{*}=\left(i_{m}\right)_{*}\left(i_{n}^{m}\right)_{*}=\left(i_{m}\right)_{*} i_{m}^{*}\left(i_{m}\right)_{*}\left(i_{n}^{m}\right)_{*}=\left(i_{m}\right)_{*} i_{m}^{*}\left(i_{n}\right)_{*} .
$$

A simplicial map $\Delta[1] \times X_{\bullet} \rightarrow Y_{\bullet}$ can be described as a collection of maps $h_{\tau}: X_{j} \rightarrow$ $Y_{j}$ for every $\tau:[j] \rightarrow[1]$, such that for $\alpha:[i] \rightarrow[j]$ one has $\alpha^{*} h_{\tau}=h_{\tau \alpha} \alpha^{*}$. A simplicial homotopy between two maps $f, g: X_{\bullet} \rightarrow Y_{\bullet}$ is a simplicial map $\Delta[1] \times X_{\bullet} \rightarrow Y_{\bullet}$ such that $h_{c_{0}^{j}}=f_{j}$ and $h_{c_{1}^{j}}=g_{j}$, where $c_{\epsilon}^{j}:[j] \rightarrow[1]$ is the constant map to $\epsilon \in[1]$.

Lemma 2.1. [16, Lemma 3.0.2.4] Let $f: A_{\bullet} \rightarrow B_{\bullet}$ and $g: B_{\bullet} \rightarrow A_{\bullet}$ be maps of simplicial schemes such that

1. $f_{m}: A_{m} \rightarrow B_{m}$ is inverse to $g_{m}$ for $m<n$;

2. $g_{n}$ is a section to $f_{n}: A_{n} \rightarrow B_{n}$;

3. $A_{\bullet} \cong \operatorname{cosk}_{n} A_{\bullet}$, and $B_{\bullet} \cong \operatorname{cosk}_{n} B_{\bullet}$.

Then $f$ and $g$ are simplicial homotopy inverse to each other.

Proof. It suffices to prove that two simplicial maps $f, g: X \bullet \rightarrow Y_{\bullet}$ on $n$-truncated schemes that are equal after $(n-1)$-truncation induce homotopic maps on the $\operatorname{cosk}_{n}$. For $i \leqslant n$ and $\tau:[i] \rightarrow[1]$, we define $h_{\tau}=f_{i}$ if $\tau=c_{0}$ and $h_{\tau}=g_{i}$ otherwise. It easily follows from the first condition that for $i, j \leqslant n$ and $\alpha:[i] \rightarrow[j]$ we have $\alpha^{*} h_{\tau}=$ $h_{\tau \alpha} \alpha^{*}$. In degrees $m>n$ we define the map $h_{\tau}$ for $\tau:[m] \rightarrow[1]$ by the following diagram



where $X_{\phi}=X_{i}$ for $\phi:[i] \rightarrow[m]$. The maps $h_{\tau \phi}: X_{i} \rightarrow Y_{i}$ are compatible with the inverse system because for $\alpha:[j] \rightarrow[i]$ we have $\alpha^{*} h_{\tau \phi}=h_{\tau \phi \alpha} \alpha^{*}: X_{i} \rightarrow Y_{j}$ by definition of $h$. It is now easy to see that this induces a map of simplicial objects; i.e., that it is compatible with the maps induced by $[q] \rightarrow[m]$. On the other hand, taking $h_{c_{0}^{m}}$ we recover $\left(\operatorname{cosk}_{n} f\right)_{m}$, and taking $h_{c_{1}^{m}}$ we recover $\left(\operatorname{cosk}_{n} g\right)_{m}$. Indeed, the maps $h_{\tau \phi}$ between inverse systems will be the system of maps $f_{i}$ and $g_{i}$, respectively.

We will apply this in combination with the following lemma:

Lemma 2.2. [16, Lemma 3.0.2.3] If $f, g: X_{\bullet} \rightarrow Y_{\bullet}$ are simplicial homotopic and $\mathcal{F}$ is a functor to an abelian category, then $\mathcal{F}(f)$ and $\mathcal{F}(g)$ are homotopic maps of the associated chain complexes.

We apply the above mostly to the category of schemes over a fixed scheme $X$. If we want to emphasize the dependence on the base scheme $X$, we write $\operatorname{cosk}_{n}\left(X_{\bullet} / X\right)$.

We often identify simplicial or multi-simplicial objects $A_{\bullet}$ in an abelian category with its corresponding chain complex without notice. 
By [18, Lemma 5.8], every proper cdh-cover can be dominated by a composition of abstract blow-ups. The same argument works under the hypothesis of the following proposition:

Proposition 2.3. Every proper ldh-cover $Y \rightarrow X$ of a separated noetherian scheme of finite Krull dimension can be dominated by a composition $S \rightarrow T \rightarrow X$, where $S \rightarrow T$ is a finite flat map of degree prime to $l$, and $T \rightarrow X$ a composition of abstract blow-ups.

Proof. (see [5], [12, Prop. 2.4]) We proceed by induction on the dimension of $X$. Basechanging with a proper cdh-cover, we can assume that $X$ is reduced and integral. Let $\eta$ be a point of $Y$ that maps to the generic point of $X$ and such that $[k(\eta): k(X)]$ is prime to $l$. Let $\tilde{Y}$ be the closure of $\eta$ in $Y$; $\tilde{Y}$ is generically finite of degree prime to $l$ over $X$. By the platification theorem [15], there is a blow-up $X^{\prime} \rightarrow X$ with center $Z$ of smaller dimension such that the strict transform $Y^{\prime} \rightarrow X^{\prime}$ of $\tilde{Y} \rightarrow X$ is flat, hence finite flat surjective of degree prime to $l$. The induction hypothesis applied to the base change $Y \times_{X} Z \rightarrow Z$ gives a factorization $S^{\prime} \rightarrow T^{\prime} \rightarrow Z$ whose union with $Y^{\prime} \rightarrow X^{\prime} \rightarrow X$ is a factorization as required.

\section{Proof of the main theorem}

\section{1. Čech covers}

We assume that $\mathcal{F}$ satisfies the hypothesis of Theorem 1.1 or 1.2.

Proposition 3.1. Let $f: X_{0} \rightarrow X$ be a proper cdh-covering. Then the augmentation map $\operatorname{cosk}_{0}\left(X_{0} / X\right) \rightarrow X$ induces a quasi-isomorphism on $\mathcal{F}(-)$. The same holds for proper ldh-coverings if $\mathcal{F}$ is a sheaf of $\mathbb{Z}_{(l)}$-modules.

Proof. We give the proof for the ldh-case and complexes of $\mathbb{Z}_{(l)}$-modules, the cdh-case follows by erasing part (b) of the proof.

(a) Given an abstract blow-up square (2), if the statement of the proposition holds for the pull-back to $Z^{\prime}, X^{\prime}$ and $Z$, then it also holds for $X$. Indeed, we obtain proper ldh-coverings $Z_{0}=Z \times_{X} X_{0} \rightarrow Z, Z_{0}^{\prime}=Z^{\prime} \times_{X} X_{0} \rightarrow Z^{\prime}$, and $X_{0}^{\prime}=X^{\prime} \times_{X} X_{0} \rightarrow X^{\prime}$. Since the functor $\operatorname{cosk}_{n}$ commutes with fiber products, we obtain on each level an abstract blow-up square upon applying the coskeleton functor. Thus we obtain a map of distinguished triangles



and if two maps are quasi-isomorphisms then so is the third.

(b) If $p: X^{\prime} \rightarrow X$ is finite flat of degree $d$ prime to $l$, and if the statement of the theorem holds for the pull-back to $X^{\prime}$, then it also holds for $X$. Indeed consider 
the diagram

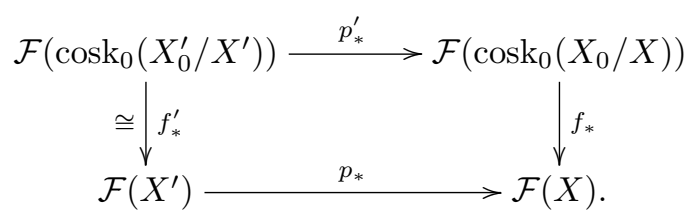

By hypothesis, $p_{*} p^{*}$ induces multiplication by the invertible number $d$ on homology, hence $p_{*}$ is split surjective on homology. Since $\operatorname{cosk}_{0}\left(X_{0} / X\right) \times_{X} X^{\prime} \cong$ $\operatorname{cosk}_{0}\left(X_{0}^{\prime} / X^{\prime}\right)$, the pull-back along $p$ is compatible with all the simplicial structure maps, hence compatibly split by $\frac{1}{d} p_{*}$ on each level. This implies that $f_{*}^{\prime}\left(p^{\prime}\right)^{*}=p^{*} f_{*}$, and that $p_{*}^{\prime}\left(p^{\prime}\right)^{*}$ induces multiplication by the invertible number $d$ on homology, so that $\left(p^{\prime}\right)^{*}$ is split injective on homology. Finally, since $p_{*} f_{*}^{\prime}=f_{*} p_{*}^{\prime}$ is surjective on homology, so is $f_{*}$, and since $f_{*}^{\prime}\left(p^{\prime}\right)^{*}=p^{*} f_{*}$ is injective on homology, so is $f_{*}$.

(c) If $f$ has a section $s: X \rightarrow X_{0}$, then the proposition follows using the contracting homotopy $s \times$ id: $X_{0}^{\times p} \rightarrow X_{0}^{\times p+1}$, where the fiber product is taken over $X$.

(d) In general, by Prop. 2.3, we can dominate $X_{0} \rightarrow X$ by a sequence $X^{\prime} \rightarrow X$ of abstract blow-ups and finite flat maps of degree prime to $l$. By (a) and (b) and induction of the dimension, it suffices to prove the theorem after base change to $X^{\prime}$. But then the map $X_{0} \times_{X} X^{\prime} \rightarrow X^{\prime}$ has a section induced by the map $X^{\prime} \rightarrow X_{0}$.

\subsection{The general case}

We now give the proof of Theorem 1.1 and Theorem 1.2 in the spirit of SGA 4 Vbis $\S 3$. Given the hyperenvelope or $l$-hyperenvelope $X_{\bullet} \rightarrow X$, let $X_{\bullet}^{n}=\operatorname{cosk}_{n} X_{\bullet}$ and consider the sequence

$$
X \bullet \stackrel{u_{n}}{\longrightarrow} X_{\bullet}^{n} \stackrel{v_{n}}{\longrightarrow} X_{\bullet}^{n-1} \stackrel{v_{n-1}}{\longrightarrow} \cdots \stackrel{v_{2}}{\longrightarrow} X_{\bullet}^{1} \stackrel{v_{1}}{\longrightarrow} X_{\bullet}^{0} \stackrel{v_{0}}{\longrightarrow} X .
$$

The map $u_{n}$ is an isomorphism in degrees $\leqslant n$, so by boundedness of $\mathcal{F}(-)$ it suffices to show that the maps $v_{n}$ induce quasi-isomorphisms on $\mathcal{F}(-)$. The case $v_{0}$ is Proposition 3.1. By (4), $v_{n}$ satisfies the conditions of the following proposition for $n \geqslant 1$ :

Proposition 3.2. [16, lemma 3.3.3.2] Let $f: K_{\bullet} \rightarrow L_{\bullet}$ be a map of simplicial schemes such that

1. $K_{p} \rightarrow L_{p}$ is an isomorphism for $p<n$;

2. $K_{n} \rightarrow L_{n}$ is a proper cdh-covering (resp. ldh-covering);

3. $K_{\bullet} \cong \operatorname{cosk}_{n} K_{\bullet}$ and $L_{\bullet} \cong \operatorname{cosk}_{n} L_{\bullet}$.

Then $\mathcal{F}\left(K_{\bullet}\right) \rightarrow \mathcal{F}\left(L_{\bullet}\right)$ is a quasi-isomorphism.

Proof. (see [2, Theorem 7.17]) Let $[K / L]_{\bullet}^{p}$ be the $p$ th fiber product of $K_{\bullet}$ over $L_{\bullet}$. Consider the bisimplicial scheme $Z_{\bullet}, \bullet$ with the $(q+1)$-fold fiber product $Z_{p, q}=$ $K_{p} \times_{L_{p}} \cdots \times_{L_{p}} K_{p}$ in bidegree $(p, q)$ such that the $p$ th column $Z_{p, \bullet}$ is $\operatorname{cosk}_{0}\left(K_{p} / L_{p}\right)$, and the $q$ th row is $Z_{\bullet, q}$ is $[K / L]_{\bullet}^{q+1}$. In particular, the $p$ th column is $K_{p}$ for $p<n$ by 
hypothesis. We have the vertical augmentation $\tilde{f}: Z_{\bullet, \bullet} \rightarrow L_{\bullet}$ induced by $f: Z_{\bullet}, 0=$ $K_{\bullet} \rightarrow L_{\bullet}$.

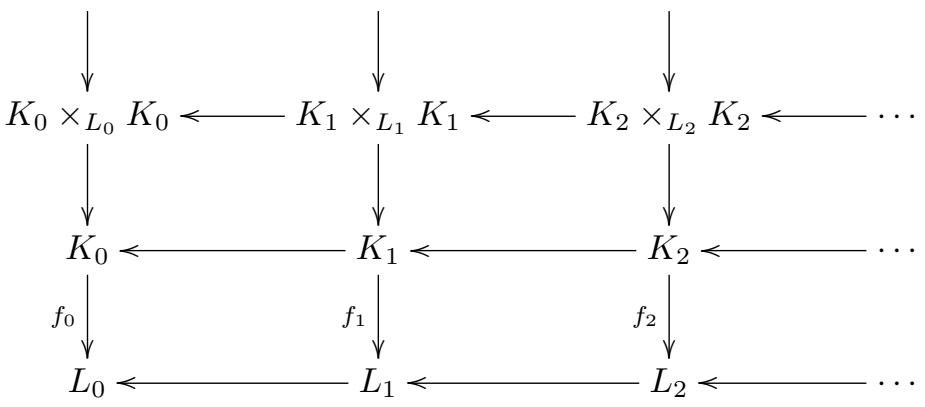

By Proposition 3.1 and the following lemma, we can see column by column that $\tilde{f}$ induces a quasi-isomorphism on $\mathcal{F}(-)$ :

Lemma 3.3. [16, Lemma 3.3.3.3] Under the assumptions of Prop. 3.2, all maps $K_{m} \rightarrow L_{m}$ are proper cdh-coverings (resp. ldh-coverings).

Proof. By (3), we have a map of equalizers:



where $D$ runs through the injections $\phi:[i] \rightarrow[m], i \leqslant n$ and $K_{\phi}:=K_{i}, L_{\phi}:=L_{i}$. It suffices to show that the fiber product of the left square $P=L_{m} \times \prod L_{\phi} \prod K_{\phi}$ has the universal property of the equalizer, because then $K_{m} \rightarrow L_{m}$ is a base-change of a proper cdh-covering (ldh-covering), hence is itself a proper cdh-cover (ldh-covering).

Given a map $u: T \rightarrow \prod_{\phi \in D_{m}} K_{\phi}$ with $s u=t u$, we have to show that there is a unique map $T \rightarrow P$ such that composition with the projection $P \rightarrow \prod_{\phi \in D_{m}} K_{\phi}$ is $u$. By definition of $L_{m}$ and $P$, it suffices to show that $s f u=t f u$, or alternatively that $f s u=f t u$, and we can do this factor by factor. Given $\alpha:[i] \rightarrow\left[i^{\prime}\right]$, there are two cases: If $i<n$, then the two maps agree because $K_{i} \rightarrow L_{i}$ is an isomorphism by hypothesis. If $i=n$, then also $i^{\prime}=n$ and $\alpha$ is the identity, hence $s=t$ trivially.

We apply Lemma 2.1 to $f$, any of the face (i.e., projection) maps $[K / L]_{\bullet}^{p+1} \rightarrow$ $[K / L]_{\bullet}^{p}$, and $g$, a degeneracy (i.e., diagonal) map that is a section of this face map. Note that the hypotheses $K_{\bullet} \cong \operatorname{cosk}_{n} K_{\bullet}$ and $L_{\bullet} \cong \operatorname{cosk}_{n} L_{\bullet}$ are preserved under fiber products ( since $\operatorname{cosk}_{n}$ is a right adjoint), hence $[K / L]_{\bullet}^{p} \cong \operatorname{cosk}_{n}[K / L]_{\bullet}^{p}$. Lemma 2.1 implies that all face maps $[K / L]_{\bullet}^{p+1} \rightarrow[K / L]_{\bullet}^{p}$ induce quasi-isomorphisms on $\mathcal{F}(-)$ that are equal on homology; hence, taking alternating sum of projection maps, we see that the maps between rows of $Z_{\bullet}, \bullet$ induce alternatingly the zero map on homology and quasi-isomorphisms on $\mathcal{F}(-)$. This implies that the inclusion $K_{\bullet} \rightarrow Z_{\bullet}, \bullet$ as the 0th row induces a quasi-isomorphism on $\mathcal{F}(-)$, hence so does the composition

$$
f: K_{\bullet} \rightarrow Z_{\bullet}, \stackrel{\tilde{f}}{\rightarrow} L_{\bullet} .
$$




\section{Applications}

\subsection{Existence of l-hyperenvelopes}

For $X$ a noetherian scheme, and $l$ a prime number, a morphism $h: X^{\prime} \rightarrow X$ is called an $l$-alteration if $h$ is proper, surjective, generically finite, sends each maximal point to a maximal point, and the degrees of the residual extensions $k\left(x^{\prime}\right) / k(x)$ over each maximal point $x$ of $X$ are prime to $l$.

Theorem 4.1. [10, Theorem X.2.1] (Gabber). Let $k$ be a field, $l$ a prime number different from the characteristic of $k, X$ separated and of finite type over $k$. Then there exists a finite extension $k^{\prime}$ of $k$ of degree prime to $l$, and a projective $l$-alteration $h: \tilde{X} \rightarrow X$ above Spec $k^{\prime} \rightarrow \operatorname{Spec} k$, such that $\tilde{X}$ is smooth and quasiprojective over $k^{\prime}$.

Corollary 4.2. In the situation of the theorem, there is a proper ldh-cover over $\tilde{X} \rightarrow$ $X$ with $\tilde{X}$ regular, and quasi-projective and of finite type over $k$.

Proof. Covering $X$ by its reduced irreducible components, we can assume that $X$ is integral and proceed by noetherian induction. Let $\tilde{X}$ be as in the theorem, and take $X^{\prime}$ the closure of a point of $\tilde{X}$ mapping to the generic point of $X$ such that the degree of the residue extension is finite prime to $l$. Let $Z$ be the closed subscheme where $X^{\prime} \rightarrow X$ is not flat. Then by induction hypothesis we can find a proper ldh-cover $Z^{\prime} \rightarrow Z$ with $Z^{\prime}$ regular, and $Z^{\prime} \amalg X^{\prime} \rightarrow X$ is the required ldh-covering.

To prove Theorem 1.5, we apply the method of $[\mathbf{3}, 6.2 .5]$ to the Corollary.

\subsection{Motivic theories}

Let $k$ be a perfect field. For a presheaf with transfers $\mathcal{P}$ on $\mathrm{Sm} / k$, recall that $\underline{\mathrm{C}}_{*}(\mathcal{P})$ is the complex of presheaves with transfers given by $\underline{\mathrm{C}}_{i}(\mathcal{P})(U)=\mathcal{P}\left(U \times \Delta^{i}\right)$ and boundary maps given by the alternating sum of pull-back maps along embeddings of faces. The complex of abelian groups $\underline{\mathrm{C}}_{*}(\mathcal{P})(k)$ is denoted by $C_{*}(\mathcal{P})$.

Recall that the cdh-topology is the coarsest Grothendieck topology generated by Nisnevich covers and proper cdh-covers, and the ldh-topology is the coarsest Grothendieck topology generated by cdh-covers and finite flat maps of degree prime to $l$.

Theorem 4.3. (1) Let $\mathcal{P}$ be a presheaf with transfers such that $\mathcal{P}_{c d h}=0$. Then under resolution of singularities, the complex of Nisnevich sheaves with transfers $\underline{C}_{*}(\mathcal{P})_{N i s}$ is acyclic.

(2) If $\mathcal{P}$ is a presheaf of $\mathbb{Z}_{(l)}$-modules with transfers such that $\mathcal{P}_{l d h}=0$, then $\underline{C}_{*}(\mathcal{P})_{N i s}$ is acyclic.

Proof. This is [4, Theorem 5.5(2)], and its extension by Kelly [11, Thm. 5.3.1]. In loc. cit. $\mathcal{P}$ is supposed to be a presheaf with transfers on all schemes, but replacing $\mathcal{P}$ by its left Kan extension to all schemes does not change $\mathcal{P}$ on smooth schemes. Moreover, loc. cit. assumes that $\mathcal{P}_{c d h}=0$, but in fact for any presheaf with transfers $\mathcal{P}_{l d h} \otimes \mathbb{Z}_{(l)}=\mathcal{P}_{c d h} \otimes \mathbb{Z}_{(l)}$ by loc. cit. Cor. 3.4.12.

Recall that a morphism $f: X \rightarrow Y$ is called equidimensional of relative dimension $r$ if it is of finite type, if every irreducible component of $X$ dominates an irreducible 
component of $Y$, and if $\operatorname{dim}_{x}\left(p^{-1} p(x)\right)=r$ for every point $x \in X$. A morphism is equidimensional if and only if it can be Zariski locally factored as $X \rightarrow \mathbb{A}_{Y}^{r} \rightarrow Y$, with the first map quasi-finite and dominant on each irreducible component of the source. A useful criterion is that a flat morphism of finite type is equidimensional of dimension $r$ if all irreducible components of all generic fibers have dimension $r$. For any scheme $X$ separated and of finite type over $k$, let $z_{\text {equi }}(X, r)$ be the presheaf with transfers on $\mathrm{Sm} / k$ which associates to $U$ the free abelian group on those closed integral subschemes of $U \times X$ which are equidimensional of relative dimension $r$ over $U$, and $c_{\text {equi }}(X, 0)$ the subpresheaf with transfers of $z_{\text {equi }}(X, 0)$ generated by those subschemes which are finite over $U$.

Proposition 4.4. For any scheme $W$ separated and of finite type over $k$, the functors $C_{*}\left(z_{\text {equi }}(-\times W, r)\right)$ and $C_{*}\left(c_{\text {equi }}(-\times W, 0)\right)$ satisfy the hypothesis of Theorems 1.1, and of Theorem 1.2 after tensoring with $\mathbb{Z}_{(l)}$.

Proof. A finite flat map $f: X \rightarrow Y$ induces a pull-back map of cycles such that the composition with push-forward is multiplication by the degree, and which is compatible with base change. To show the exactness of the triangle resulting from an abstract blow-up, it suffices by Theorem 4.3 to show that the functors $\mathcal{P}: X \mapsto z_{\text {equi }}(X \times W, r)$ and $\mathcal{P}: X \mapsto c_{\text {equi }}(-\times W, 0)$ (tensored with $\mathbb{Z}_{(l)}$ in the case of Theorem 1.2) send abstract blow-up squares to short exact sequences of ldh-sheaves (or cdh-sheaves under resolution of singularities). Replacing $X, X^{\prime}, Z, Z^{\prime}$ by their product with $W$, we can drop $W$ from the notation. Only the surjectivity of $\mathcal{P}\left(X^{\prime}\right) \oplus \mathcal{P}(Z) \rightarrow \mathcal{P}(X)$ is difficult.

We repeat the platification argument of Suslin-Voevodsky [18, Theorem 4.7] and Friedlander-Voevodsky [4, Theorem 5.11]. Given a section $S \in \mathcal{P}(X)(U)$, we need to find a cdh-covering (ldh-covering) $V \rightarrow U$ such that $\left.S\right|_{V}$ is in the image of $\mathcal{P}\left(X^{\prime}\right)(V) \oplus$ $\mathcal{P}(Z)(V)$. This is clear if $S \subseteq U \times Z$. Otherwise let $T$ be the closure of $S \cap U \times$ $(X-Z) \subseteq U \times(X-Z) \cong U \times\left(X^{\prime}-X^{\prime} \times{ }_{X} Z\right)$ in $U \times X^{\prime}$.

Then $T$ may not be equidimensional, but by the platification theorem [15], we can find a blow-up $U^{\prime} \rightarrow U$ such that the proper transform $T^{\prime}$ of $T$ in $U^{\prime} \times X^{\prime}$ is flat over $U^{\prime}$. By Corollary 4.2, we can find a cdh-cover (respectively ldh-cover) $V \rightarrow U^{\prime}$ with $V$ smooth, and we let $T^{*}$ be the pull-back of $T^{\prime}$ to $V$. Then $T^{*} \rightarrow V$ is flat, hence equidimensional.

Friedlander and Voevodsky $[4, \S 9$, Def. 4.3$]$ define Borel-Moore homology of $X$ to be

$$
H_{i}^{B M}(X, A(n))= \begin{cases}H_{i-2 n} C_{*}\left(z_{\text {equi }}(X, n) \otimes A\right) & n \geqslant 0 \\ H_{i-2 n} C_{*}\left(z_{\text {equi }}\left(X \times \mathbb{A}^{-n}, 0\right) \otimes A\right) & n<0,\end{cases}
$$

and motivic homology to be

$$
H_{i}(X, A(n))= \begin{cases}H_{\{0\}}^{2 n-i}\left(\mathbb{A}^{n}, \underline{C}_{*}\left(c_{\text {equi }}(X, 0) \otimes A\right)\right) & n \geqslant 0 \\ H_{i-2 n-1} C_{*}\left(\frac{c_{\text {equi }}\left(X \times\left(\mathbb{A}^{-n}-\{0\}\right), 0\right)}{c_{\text {equi }}(X \times\{1\}, 0)} \otimes A\right) & n<0 .\end{cases}
$$

Proof of Theorem 1.3: For Borel-Moore homology, the theorem follows from Prop. 4.4. For homology in negative weights, the theorem follows because the inclusion $c_{\text {equi }}(X \times\{1\}, 0) \rightarrow c_{\text {equi }}\left(X \times\left(\mathbb{A}^{-n}-\{0\}\right), 0\right)$ is canonically split by the structure map, so that the conclusion of Proposition 4.4 applies to this situation. 
Finally, motivic homology in positive weights is the homology of the cone of the split injection

$$
C_{*}\left(c_{\text {equi }}(X, 0)\right) \otimes A \rightarrow R \Gamma\left(\mathbb{A}^{n}, \underline{C}_{*}\left(c_{\text {equi }}(X, 0) \otimes A\right)\right) .
$$

Consider the Godement resolution for the Nisnevich topology of the affine space. We can bound it, because the cohomological dimension of $\mathbb{A}^{n}$ is $n$. Furthermore, by $[\mathbf{1 4}$, Example 6.20], the terms of this resolution are still presheaves with transfers. Since the Godement resolution is functorial and sends short exact sequences of sheaves to short exact sequences of sheaves, it inherits the hypothesis of Theorems 1.1 and 1.2 from $c_{\text {equi }}(X, 0)$.

Proof of Theorem 1.4: This follows easily from the localization property of higher Chow groups $[\mathbf{1}, \mathbf{1 3}]$.

\subsection{Other applications}

In [6] we show that Parshin's conjecture implies that for a smooth variety over a finite field, higher Suslin homology vanishes rationally; i.e., $H_{p}^{S}(Y, \mathbb{Q})=0$ for smooth $Y$ and $p>0$. This implies that for every $l$-hyperenvelope consisting of smooth schemes there is an isomorphism

$$
H_{p}^{S}(X, \mathbb{Q}) \cong H_{p}\left(H_{0}^{S}\left(X_{\bullet}, \mathbb{Q}\right)\right) .
$$

In [7], we use Corollary 1.3 to show that for a normal connected variety $X$ over an algebraically closed field, the Albanese map

$$
a l b_{X}: H_{0}(X, \mathbb{Z})^{0} \rightarrow \operatorname{Alb}_{X}(k)
$$

is an isomorphism on torsion groups away from the characteristic, and at the characteristic under resolution of singularities.

\section{References}

[1] Bloch, S., Algebraic cycles and higher K-theory, Adv. in Math. 61 (1986), no. 3, 267-304.

[2] Conrad, B., Cohomological descent, preprint, 2003.

[3] Deligne, P., Theorie de Hodge III, Inst. Hautes Etudes Sci. Publ. Math. 44 (1974), 5-77.

[4] Friedlander, E. and Voevodsky, V., Bivariant cycle homology. Cycles, transfers, and motivic homology theories, 138-187, Ann. of Math. Stud. 143, Princeton Univ. Press, Princeton, NJ, 2000.

[5] Geisser, T., Arithmetic cohomology over finite fields and special values of ל-functions, Duke Math. J. 133 (2006), no. 1, 27-57.

[6] Geisser, T., On Suslin's singular homology and cohomology, Doc. Math. (2010), extra volume: Andrei A. Suslin sixtieth birthday, 223-249.

[7] Geisser, T., Rojtman's theorem for normal schemes, preprint, 2014, available at http://arxiv.org/abs/1402.1831.

[8] Gillet, H., Homological descent for the K-theory of coherent sheaves. Algebraic K-theory, number theory, geometry and analysis (Bielefeld, 1982), 80-103, Lecture Notes in Math. 1046, Springer, Berlin, 1984. 
[9] Gillet, H. and Soulé, C., Motivic weight complexes for arithmetic varieties, J. Algebra 322 (2009), no. 9, 3088-3141.

[10] Illusie, L., Laszlo, Y., and Orgogozo, F., Travaux de Gabber sur l'uniformisation locale et la cohomologie étale des schémas quasi-excellents. Séminarie à l’École polytechnique 2006-2008.

[11] Kelly, S., Triangulated categories of motives in positive characteristic, preprint, 2013, available at http://arxiv.org/abs/1305.5349.

[12] Kelly, S., Vanishing of negative $K$-theory in positive characteristic, preprint, 2011, available at http://arxiv.org/abs/1112.5206v4.

[13] Levine, M., Techniques of localization in the theory of algebraic cycles, J. Algebraic Geom. 10 (2001), no. 2, 299-363.

[14] Mazza, C., Voevodsky, V., and Weibel, C., Lecture notes on motivic cohomology. Clay Mathematics Monographs, 2. American Mathematical Society, Providence, RI; Clay Mathematics Institute, Cambridge, MA, 2006.

[15] Raynaud, M. and Gruson, L., Criteres de platitude et de projectivite. Techniques de "platification" d'un module, Invent. Math. 13 (1971), 1-89.

[16] Saint-Donat, B., Techniques de descentes cohomologique, in: Artin, Michael, Grothendieck, Alexandre, Verdier, J.K, Theorie des topos et cohomologie etale des schemas. Lecture Notes in Mathematics, Vol. 270. Springer-Verlag, BerlinNew York, 1972.

[17] Suslin, A. and Voevodsky, V., Singular homology of abstract algebraic varieties, Invent. Math. 123 (1996), no. 1, 61-94.

[18] Suslin, A. and Voevodsky, V., Bloch-Kato conjecture and motivic cohomology with finite coefficients. The arithmetic and geometry of algebraic cycles (Banff, AB, 1998), 117-189, NATO Sci. Ser. C Math. Phys. Sci. 548, Kluwer Acad. Publ., Dordrecht, 2000.

Thomas Geisser geisser@math.nagoya-u.ac.jp

Graduate School of Mathematics, Nagoya University, Nagoya 464-8602, Japan 